\title{
Dual-Specificity Protein Phosphatase 26 (DUSP26) Inhibits LPS-Induced TNF- $\alpha$ Production
}

\author{
Huiyun Seo and Sayeon Cho* \\ College of Pharmacy, Chung-Ang University, Seoul 156-756, Korea. ${ }^{*}$ E-mail: sycho@cau.ac.kr \\ Received July 5, 2010, Accepted July 13, 2010
}

Key Words: Lipopolysaccharide, TNF- $\alpha$, Dual specificity phosphatase 26, Mitogen-activated protein kinase

Mitogen-activated protein kinase (MAPK) pathway is a wellknown example that cellular biochemical network maintains delicate balance which is regulated during inflammatory responses. ${ }^{1}$ The MAPK subfamily, including the c-Jun N-terminal kinase (JNK), extracellular signal-regulated kinases (ERK), and p38, act as key regulatory components as inflammatory mediators in the mammalian innate immune system. ${ }^{1,2}$ As several studies have shown, phosphorylation of MAPKs in the signal transduction pathway plays critical roles on downstream events of inflammatory response. ${ }^{3}$ Innate immune cells such as macrophages stimulated with lipopolysaccharide (LPS) release proinflammatory cytokine products such as tumor necrosis factor-alpha (TNF- $\alpha$ ), interleukin-6 (IL-6), interleukin-12 (IL-12), monocyte chemotactic protein-1 (MCP-1), interferon-gamma (IFN- $\gamma$ ), and interleukin-10 (IL-10) through complex signaling mechanism. ${ }^{4,5}$ A heterogeneous group of protein phosphatases named dual specificity phosphatases (DUSPs) is known to regulate MAPK pathway by dephosphorylating both the phosphoserine/threonine and phosphotyrosine residues of individual MAPKs. ${ }^{6}$ Several groups of evidence demonstrate that this subclass of DUSPs may play roles as essential feedback regulators in the innate immune response. Exposure of RAW264.7 cells to LPS induces the expression of DUSP1 that inactivates JNK and p38 and leads to inhibition of cytokine productions. ${ }^{7}$ DUSP26, a member of atypical DUSPs, is a $24-\mathrm{kDa}$ protein that was identified using a yeast two-hybrid screen by Mivechi's research group in $2005 .^{8}$ DUSP26 has several functions as a p38 and an ERK-specific phosphatase. ${ }^{8,9}$

In this study, we examined the relationship of TNF- $\alpha$ induction and DUSP26 in LPS-stimulated RAW264.7 cells. To examine the function of DUSP26 in the regulation of TNF- $\alpha$ production, RAW264.7 cells that were transfected with FLAG-tagged DUSP26 expression vector were stimulated with LPS. TNF- $\alpha$ concentrations in the media were measured by Enzyme-linked immunosorbent assay (ELISA). Cells transfected with FLAGDUSP26 plasmid secretes less TNF- $\alpha$ after LPS stimulation than cells transfected with empty plasmid (Fig. 1). TNF- $\alpha$ secretion was further reduced when more FLAG-DUSP26 plasmid was transfected, as shown by the $90 \%$ reduction in TNF- $\alpha$ secretion. Our results indicate that increased DUSP26 expression inhibits TNF- $\alpha$ production in LPS-stimulated macrophages.

Next, DUSP26 expression levels were determined using mRNA obtained from LPS-stimulated RAW264.7 cells by RTPCR. DUSP1 expression, which was used as a control, increased after 1 h exposure of LPS to RAW264.7 cells as shown Fig. 2.
DUSP26 mRNA, however, was not changed in RAW264.7 cells treated with LPS. These results suggest that DUSP26 expression is not significantly regulated by LPS treatment.

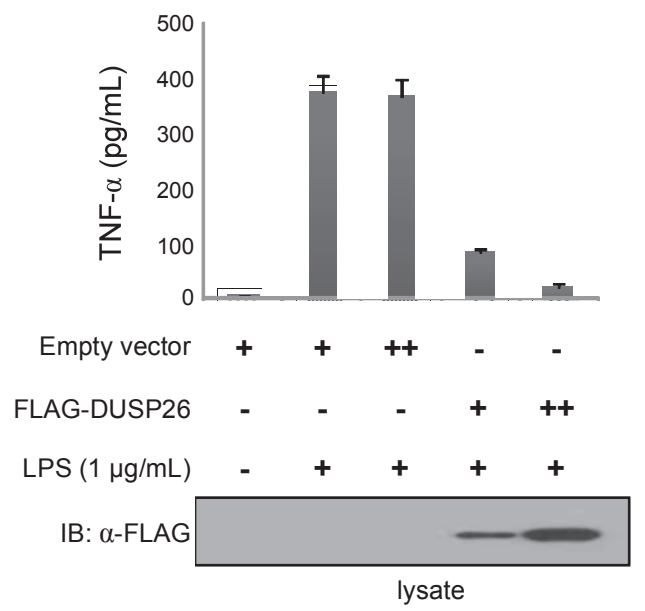

Figure 1. Overexpression of DUSP26 in macrophages inhibits LPSstimulated TNF- $\alpha$ production. RAW264.7 cells were transfected with either the empty vector or the construct expressing FLAG-DUSP26. After $16 \mathrm{~h}$ stimulation with LPS $(1 \mu \mathrm{g} / \mathrm{mL})$, supernatants were analyzed for TNF- $\alpha$ production using an ELISA assay as described in the Experimental Section. Cell lysates were subjected to immunoblotting using an anti-FLAG antibody for detection of DUSP26. The results presented are representative of three independent experiments. Error bars indicate \pm SEM.

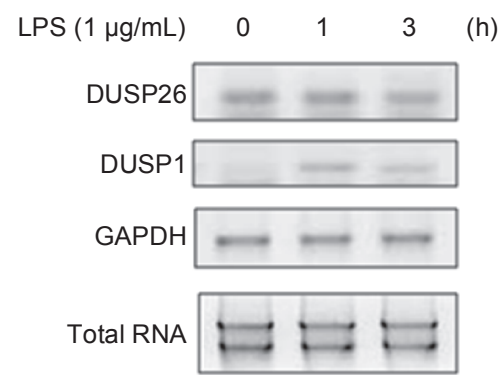

Figure 2. DUSP26 expression in RAW264.7 cells is not induced after treatment with LPS. Expression levels of DUSP26 mRNA in RAW264.7 cells were determined at the indicated times after treatment of LPS $(1 \mu \mathrm{g} / \mathrm{mL})$ by RT-PCR. GAPDH transcripts were amplified using specific primers (forward 5'-ACCACCATGGAGAAGGC-3'; reverse 5'-CTCAGTGTAGCCCAGG ATGC-3') as a control for RNA integrity. DUSP1 transcripts were amplified and used as a control for LPS treatment. 


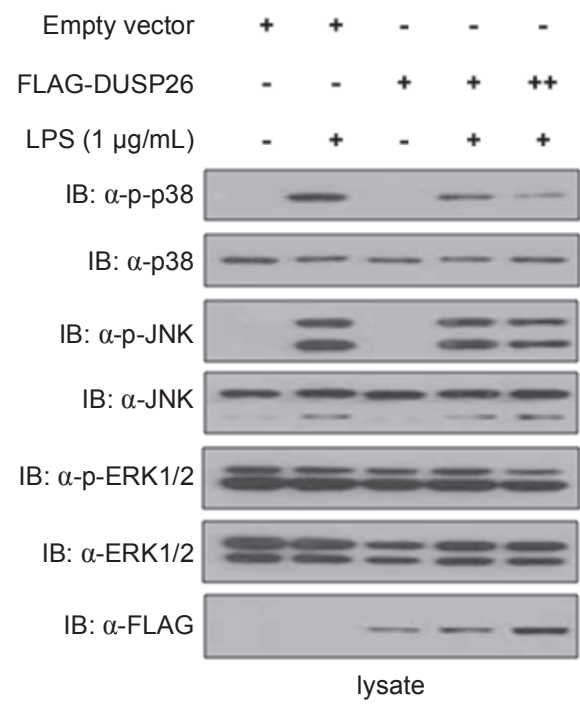

Figure 3. Effects of DUSP26 on LPS-mediated MAPK signal transduction. DUSP26 expression plasmid was transfected into RAW264.7 cells and then cells were stimulated with LPS $(1 \mu \mathrm{g} / \mathrm{mL})$ for $1 \mathrm{~h}$. Immunoblottings were performed for total and phosphorylated proteins as indicated. Three independent experiments were performed with similar results.

We next examined whether DUSP26 influences the phosphorylation level of DUSP26 substrates in RAW264.7 cells. It has been reported that MAPKs are substrates of DUSP26. However, the role of DUSP26 on MAPKs was controversial. Yu et al. reported that DUSP26 dephosphorylates and inhibits p38 ${ }^{10}$ while Hu et al. suggested that DUSP26 inactivates ERK1/2 and JNK $1 .{ }^{8}$ In another study, DUSP26 does not act as a MAPK phosphatase but as modulator of the PI3K/Akt pathway by an unknown mechanism. ${ }^{11}$ We performed immunoblotting analysis to measure the endogenous levels of phospho-JNK, phosphop38, and phospho-ERK1/2. After RAW264.7 cells were transfected with FLAG-tagged DUSP26 expression plasmids or empty plasmids for $24 \mathrm{~h}$, cells were exposed to LPS. Then, the levels of phosphorylated MAPKs were determined with immunoblotting analysis using anti-phospho-MAPK antibodies. RAW264.7 cells transfected with empty plasmids prior to LPS treatment showed enhanced activation of phospho-JNK, phospho-p38, and phospho-ERK1/2. However, RAW264.7 cells transfected with FLAG-DUSP26 expression plasmid prior to LPS treatment showed reduced phospho-p38 level without changing the expression level of p38. In addition, phospho-JNK level was slightly declined with overexpression of DUSP26 whereas phospho-ERK1/2 level was not affected (Fig. 3). Consequently, our results suggest that DUSP26 induces dephosphorylation and thus inactivation of p38 significantly and JNK slightly but not ERK1/2.

In conclusion, we found that DUSP26 acts as a negative regulator in the inflammatory response of macrophages in innate immune system. In LPS-stimulated RAW264.7 cells, DUSP26 inhibits TNF- $\alpha$ production by inactivation of $\mathrm{p} 38$ and JNK.

\section{Experimental Section}

Cell culture and transfection. The murine macrophage RAW
264.7 cells were maintained at $37{ }^{\circ} \mathrm{C}$ in Dulbecco's modified Eagle's medium (DMEM, Invitrogen, Carlsbad California) supplemented with $10 \%$ fetal bovine serum (FBS, Invitrogen) and penicillin/streptomycin in the presence of $5 \% \mathrm{CO}_{2}$. For transient transfection, $1.4 \times 10^{6}$ cells were plated in each $60-\mathrm{mm}$ cell culture plate, grown overnight, and transfected with DNA using FuGENE HD transfection reagent (Roche, Basel, Switzerland).

Plasmid constructs. The N-terminal FLAG-tagged DUSP26 for expression in mammalian cells was constructed by polymerase chain reaction (PCR), followed by cloning into the pcDNA3.1/Zeo plasmid.

Reagents and antibodies. Polyclonal anti-JNK, anti-phosphoJNK (Thr-183/Tyr-185), anti-p38, anti-phospho-p38 (Thr-180/ Tyr-182), anti-ERK1/2, and anti-phospho-ERK1/2 (Thr-202/ Tyr-204) antibodies were purchased from Cell Signaling Technology (Danvers, MA). Anti-FLAG M2 and LPS were from Sigma-Aldrich (St. Louis, MO).

TNF- $\alpha$ ELISA. TNF- $\alpha$ protein concentrations were determined by standard sandwich ELISA using antibodies and standards obtained from BD Biosciences (San Diego, CA) and used according to manufacturer's instructions. Assays were performed on neat and diluted samples in duplicate on 96-well plates. Absorbance was measured by a microplate reader at $450 \mathrm{~nm}$ and concentrations were determined by comparison to a standard curve. All transfections were repeated at least three times.

Reverse transcription-polymerase chain reaction (RT-PCR). Total RNAs were prepared from cells by Trizol (Invitrogen) and reverse transcription was performed using M-MLV Reverse Transcriptase (Invitrogen). PCR for mouse DUSP26 cDNA was carried out using the following primers: forward 5'-GCAAGA CAGCCTGTAACCATGC-3' and reverse 5'-GCACCAGGAT CTTCCCTCCTG-3'.

Immunoblotting analysis. After RAW264.7 cells were transiently transfected with FLAG-tagged DUSP26 expression plasmids or empty plasmids for $48 \mathrm{~h}$, cells were washed twice with phosphate buffered saline (PBS) buffer and lysed in PTP lysis buffer (0.5\% NP-40, 0.5\% Triton X-100, $150 \mathrm{mM} \mathrm{NaCl}$, 20 mM Tris- $\mathrm{HCl}$ (pH 8.0), 1 mM EDTA, 1\% glycerol, $1 \mathrm{mM}$ phenylmethylsulfonyl fluoride, and $1 \mu \mathrm{g} / \mathrm{mL}$ Aprotinin) for 30 $\min$ at $4{ }^{\circ} \mathrm{C}$. Cleared cell lysates from centrifugation were resuspended with protein sample buffer, boiled at $100{ }^{\circ} \mathrm{C}$ for $5 \mathrm{~min}$, subjected to SDS-PAGE, and subsequently transferred onto nitrocellulose membrane. Immunoblotting analysis was carried out as previously described. ${ }^{12}$

Acknowledgments. This work was supported by a grant of the Korea Health 21 R\&D Project, Ministry of Health \& Welfare, Republic of Korea (A010385-1033-0000400) and by the Korea Research Foundation Grant funded by the Korean Government (MOEHRD, Basic Research Promotion Fund) (KRF2008-331-E00089).

\section{References}

1. Salojin, K.; Oravecz, T. J. Leukoc. Biol. 2007, 81, 860-869.

2. Dong, C.; Davis, R. J.; Flavell, R. A. Annu. Rev. Immunol. 2002, 20, 55-72.

3. Lang, R.; Hammer, M.; Mages, J. J. Immunol. 2006, 177, 7497- 
7504.

4. Rothe, J.; Lesslauer, W.; Lotscher, H.; Lang, Y.; Koebel, P.; Kontgen, F.; Althage, A.; Zinkernagel, R.; Steinmetz, M.; Bluethmann, H. Nature 1993, 364, 798-802.

5. Salojin, K. V.; Owusu, I. B.; Millerchip, K. A.; Potter, M.; Platt, K. A.; Oravecz, T. J. Immunol. 2006, 176, 1899-1907.

6. Patterson, K. I.; Brummer, T.; O'Brien, P. M.; Daly, R. J. Biochem. J. 2009, 418, 475-489.

7. Wang, X.; Liu, Y. Cell Signal. 2007, 19, 1372-1382.
8. Hu, Y.; Mivechi, N. F. Mol. Cell. Biol. 2006, 26, 3282-3294.

9. Vasudevan, S. A.; Skoko, J.; Wang, K.; Burlingame, S. M.; Patel, P. N.; Lazo, J. S.; Nuchtern, J. G.; Yang, J. Biochem. Biophys. Res. Commun. 2005, 330, 511-518.

10. Yu, W.; Imoto, I.; Inoue, J.; Onda, M.; Emi, M.; Inazawa, J. Oncogene 2007, 26, 1178-1187.

11. Takagaki, K.; Shima, H.; Tanuma, N.; Nomura, M.; Satoh, T.; Watanabe, M.; Kikuchi, K. Mol. Cell. Biochem. 2007, 296, 177-184.

12. Song, M.; Cho, S. Bull. Korean Chem. Soc. 2009, 30, 1190-1192. 